\title{
CIRCULAR MICROSTRUCTURAL VOLUME ELEMENTS WITH PERIODIC BOUNDARY CONDITIONS FOR LOCALIZATION PROBLEMS
}

\author{
Pieter Hofman*, Lu Ke and Frans P. van der Meer \\ Delft University of Technology, Faculty of Civil Engineering and Geosciences \\ *p.hofmandstudent.tudelft.nl
}

A common choice for multiscale modelling of the mechanical response of composites is to use periodic boundary conditions (PBCs) on square representative volume elements (RVEs). Unfortunately, these PBCs over-constrain the response when strain localization takes place in bands that are not compatible with the imposed periodic constraints.

Previously developed improvements are based on aligning the PBCs with an evolving localization band. This is either done by applying a rotation to the periodicity frame or imposing the PBCs in a weak sense and apply a shift to the mapping function that couples points. However, with matrix-fiber RVEs, this shift implies a mis-alignment of fibers that cross opposing edges, which limits the number of supported localization angles [1].

This contribution explores the use of circular RVEs with PBCs to obtain a micromodel with transversely isotropic response. It is shown that the original formulation proposed in [2] fails to provide the full softening response due to over-constraining when cracks reach the boundary. Therefore, we propose a modification to the PBCs which allows for cracks to cross the edges. The performance of the model is tested with a series of simulations where a macroscopic uniaxial strain rate is imposed under varying angles. It is demonstrated that the circular RVE with the modified PBCs successfully predicts an isotropic response with full softening (see Figure 1). Furthermore, it is shown that no pathological dependence on the size of the RVE exists.
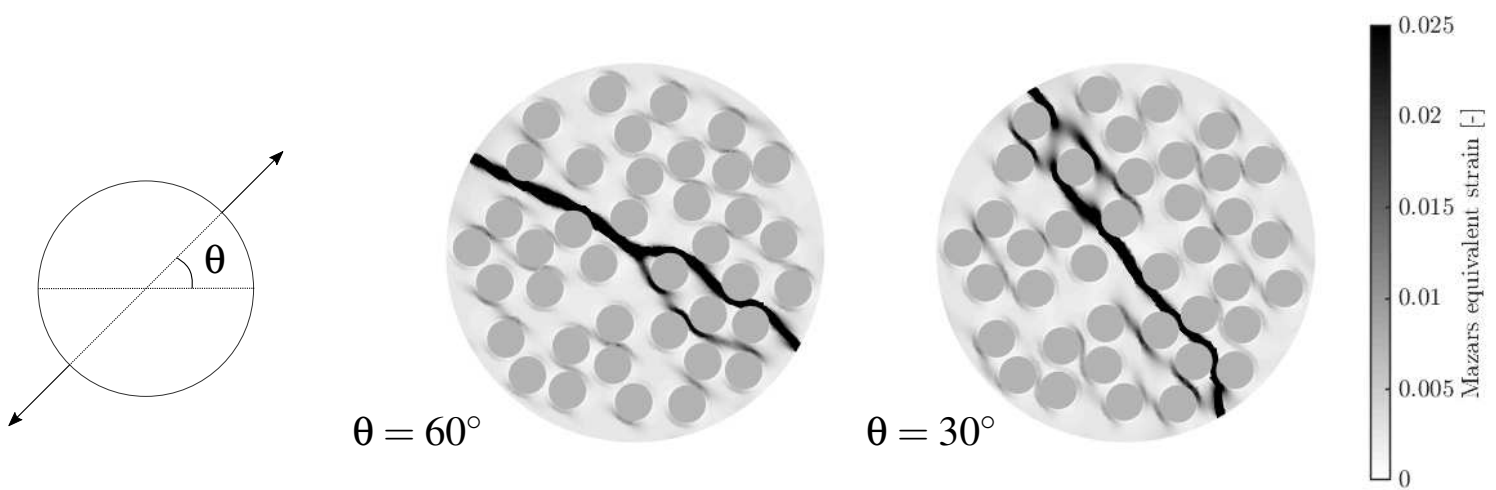

Figure 1: Inclined crack bands on the circular RVEs with the modified PBCs. A uniaxial strain is imposed under varying loading orientation angles $\theta$.

\section{References}

[1] E. Giesen Loo and F.P. van der Meer. (2020) Stress-controlled weakly periodic boundary conditions: Axial stress under varying orientations. International Journal for Numerical Methods in Engineering, 10.1002/nme.6441.

[2] R. Glüge, M. Weber, A. Bertram (2012). Comparison of spherical and cubical statistical volume elements with respect to convergence, anisotropy, and localization behavior. Computational Materials Science, 63:91104 